\title{
Changes in Lifestyle, Exercises, Possible Mechanisms and Associ- ations with Prostate Cancer
}

\section{Mauro Sergio Perilhão ${ }^{1}$, Roberta Luksevicius Rica ${ }^{2}$, Alexandre Lopes Evangelista ${ }^{3}$, Thaís Alves da Silva ${ }^{1}$, Aylton Figueria Junior ${ }^{2}$ and Danilo Sales Bocalini ${ }^{4 *}$}

\author{
${ }^{1}$ Laboratory of Exercise Genetic and Metabolism. Department of Biophysics, Federal University of São \\ Paulo, SP, Brazil \\ ${ }^{2}$ Department of Physical Education, São Judas Tadeu University, São Paulo, SP, Brazil \\ ${ }^{3}$ Departament of Education, Physical Education Division, Nove de Julho University, São Paulo, SP, Brazil \\ ${ }^{4}$ Experimental Physiology and Biochemstry Laboratory, Physical Education Sport Center, Federal University \\ of Espirito Santo, Vitoria, ES, Brazil
}

*Corresponding author: Danilo Sales Bocalini, Translational Physiology Laboratory, Sao Judas Tadeu University, 546 Taquari St, $2^{\text {nd }}$ floor, Sao Paulo, 03166-000, Brazil, Tel: 27991677, E-mail: bocaliniht@hotmail.com

\begin{abstract}
Prostate cancer is characterized by overgrowth of the prostate, followed by a decrease in the size and intensity of the urinary stream, which is considered a major cause of illness and death worldwide. Its etiology is unknown. Physical activity and sedentary lifestyles are placed in the context of lifestyle, which has been studied since researchers realized that changes in one's living influence the onset of prostate cancer. Checking if the exercise influence in prostatic neoplasia, whether there is a relationship, association and the possible mechanisms involved in the process. In 54 studies which investigated the possible associations between exercise and prostate cancer, 26 showed reverse association, 23 state that there is no association and 5 as increased risk. For patients undergoing traditional treatments, especially those deprived of androgens, aerobic, resistance, or combined exercises have been proven beneficial. The recommendations are to perform at least 20 to 30 minutes of aerobic exercise with moderate to vigorous intensity 5 times per week, as well as 2 times per week of resistance exercises for the major muscle groups, with moderate intensity. There is no confirmation of the association between exercise and prostate cancer but there is definitive evidence for this relationship. However, further studies controlling the different types of physical activities, their volumes and intensities, and which mechanisms are involved, should be conducted.
\end{abstract}

\section{Keywords}

Prostate cancer, Lifestyle, Exercise, Physical activity, Aging

\section{Introduction}

According to the World Health Organization (WHO) in Tonon, et al. [1] state prostate cancer is the most common neoplasia in men, and the second most common cause of death in Brazil.

Alongside the increase in life expectancy, as the individual approaches old age prostate cancer is becoming part of an increasingly broader scope. This cancer may be detected and treated during its early stages. The prostate is a small gland located in the male pelvis. It weighs approximately $20 \mathrm{~g}$, and it is responsible for producing $40 \%$ to $50 \%$ of the fluids which constitute the semen/sperm, providing it with protection and nutrients essential to the survival of sperm cells.

As men age, the size of the prostate increases, slowing down the urinary stream and making it harder for the body to expel urine.

According to the INCA (Instituto Nacional de Câncer) [2], Brazilian National Cancer Institute, 13,129 deaths in Brazil in 2011 were caused by prostate cancer, and an estimated 68,800 cases were likely to emerge in 2014.

Its etiopathogenesis is unknown, but there are many factors which are likely to cause its onset: Aging, hor- 
monal metabolism, lifestyle, sexual history, exposure to pathogens and to industrial chemical substances, urbanization, dietary habits, and heredity.

With the knowledge that changes in lifestyle influence the etiology of the prostatic neoplasia, and that exercising (or lack thereof) do constitute a lifestyle, it is likely that physical activity may somehow interfere with prostate cancer.

The general aim of this study is to find out if physical exercise influences prostate cancer, specifically aiming to show any relationships between exercise and cancer, and which mechanisms lead to this association.

\section{Bibliographic Search}

The bibliographic search was performed electronically using PubMed database as search engine; November 2014 was the cutoff point. The following key words: "Prostate cancer, Lifestyle, Exercise, Physical activity and Aging", were used. Instituto Nacional de Câncer (INCA) site also were consulted.

\section{Results}

\section{Lifestyle}

Schreiber [3] emphasizes the issue regarding lifestyle changes as a way to curb several types of cancer.

Studies by Frattaroli, et al. [4] show that when patients in the early stages of prostate cancer change their lifestyle, they may partially avoid or delay conventional treatment for at least 2 years (the time taken to conduct the studies).

Daubenmier, et al. [5] show that there are improvements in quality of life, physical and mental capabilities, and sexual functioning compared to the control group. Ornish, et al. [6] show changes in lifestyle, including taking 30-minute walks six times a week in moderate intensity, reduces tumoral markings and Prostatic-Specific Antigen (PSA) by $4 \%$ among the group who engaged in lifestyle changes, and by $8 \%$ in the control group. The researchers have also proved the growth of cancer cells in the prostate was inhibited eight times more than the control group. As such, lifestyle changes may positively affect low degree prostate cancer during early diagnosis.

In another study, Ornish, et al. [7] demonstrated the lack of telomeres, prognostic markers of aging, diseases and premature morbidity, in humans. Within three months of lifestyle changes, the researchers detected an increase in telomerase in immune system cells.

Several authors, including Antonelli, et al. [8], Khan, et al. [9], Sheffield, et al. [10], Culos-Reed, et al. [11], Schneider, et al. [12] and Blanchard, et al. [13] also describe the importance of lifestyle changes in improving the quality of life of prostate cancer patients.

Khan, et al. [9] show the occurrence of prostate cancer varies greatly depending on geographical region.
Asia, for instance, has low mortality rates and low risks of death when dealing with this cancer, but the risk is higher in Europe and the United States. Occurrence increases significantly in immigrant groups from Asia to North America, indicating that it is very likely that certain lifestyles might be the main causes of death in prostate cancer cases.

\section{Exercise and the likely associations with prostate cancer}

Certain studies analyze the existence of an association between exercise and prevention, delay, progression, and decrease of prostate cancer risks.

Antonelli, et al. [8] in an extensive review, state no research established the effects of exercise as a type of monotherapy for prostate cancer, but the molecular mechanisms responsible for the likely association between exercise and prostate cancer are being clarified. It will then allow researchers to find out the role of exercise in preventing or delaying the pathogenesis of prostate cancer.

In 32 reviewed studies which set out to investigate the association between prostate cancer and exercise, more than half of these studies show a reverse relation, while others showed association, including four which show an increase in risk.

In accordance with that, Johnsen, et al. [14] researched 22 cohort studies. Of these studies, nine showed a reverse association between the risk of prostate cancer and physical aptitude, occupational activity, and leisurely activities. One study showed increased risk in university athletes, while 12 showed no associations.

A new reported from a group of specialists of the World Cancer Research Foundation and the American Institute for Cancer Research cited by Antonelli, et al. [8] concluded that evidence supporting the association between exercise and prostate cancer is restricted to formal judging. In this same article, another major prospective cohort study involving 23 locations in 10 European countries was conducted to verify dietary habits, lifestyle, and occurrence of different types of cancer, revealing a tendency towards reduced risk of prostate cancer in individuals engaging in higher physical occupation. One particularly notable tendency was towards advanced prostate cancer, and researchers concluded that high-level occupational physical activities may delay its progression.

Moore, et al. [15] demonstrated a seven-year study involving 160,006 white male and 3,671 black males between the ages of 51 and 72 , using a questionnaire, with physical activity levels between 15 and 18 years, 19 and 29 years, 35 and 39 years, and the last 10 years. It was categorized by intensity (low, moderate, vigorous, and total) in relation to the risk of prostate cancer. It was found 9,624 white male and 371 black male developed prostate cancer. Among the white male, there was no 
association with physical activity, regardless of the period between ages and the intensity of exercises. Black male, however, who engaged in four hours of moderate to vigorous physical activity or more, displayed $35 \%$ less risks compared to the group which rarely exercised, during period of 19 to 29 years of age.

For frequent physical activity between the ages of 35 and 39 , there was a tendency towards reduced risk. In this study, volumes and intensities were divided as follows: Three METs for low physical activity, five METs for moderate. Regularity included: Never, rarely, one hour a week, one to three hours a week, four to seven hours a week, and more than seven hours a week.

They further reference three studies involving black male with prostate cancer, one of which associated lack of physical activity with three times the likelihood of the onset of prostate cancer. The other two studies were void.

Richman, et al. [16] mention a decrease of $61 \%$ of the mortality risk of metastatic prostate cancer regarding three times a week or more of physical activity, compared to the group engaged in less than an hour a week. Physical activity (walking) was considered vigorous for six METs or more. Men who walked three hours a week or more in a more intense pace had a statistically significant reduction rate of $57 \%$ towards the tumor's progression compared to the less-than-three-hour/lower-intensity-pace group. They further mention a study which verified that a quick-paced walk (classified as "Somewhat Hard" in the Borg Rating of Perceived Exertion Scale) may affect the progression of prostate cancer in elderly male, reducing inflammation. The researchers have also reported reduced levels of interleukina-6, which may cause the proliferation of cancer cells, as well as inhibiting their apoptosis in in vitro prostate. High levels of interleukina- 6 involve prostate cancer may lead to an increased risk of death by $73 \%$.

Shahar, et al. [17] consider physical activity a global health issue, and that the risk of prostate cancer decreased between those who engaged in arduous physical activity in early adulthood. In yet another study, researchers state the risk of metastatic prostate cancer is reduced when associated with higher levels of physical activity. They mention four mechanisms that could explain the influence of physical activity in reducing the risk of prostate cancer: Active male may have lower levels of androgynous hormones, less body fat, improved immune function, and superior antioxidant defense mechanism.

Liu, et al. [18] concluded that there appears to be a reverse association between physical activity and the risk of prostate cancer, as well as countless other health benefits, in a meta-analysis involving 88,204 cases, 16 eligible cohort studies, and 24 eligible control case studies.
Heitkamp, et al. [19] agree that intense physical activity may contribute to preventing prostate carcinoma.

Crespo, et al. [20] however, concluded that exercise did not prevent prostate cancer mortality in a group of Puerto Ricans.

Certain studies on mice also tried to show likely associations between physical activity and prostatic carcinoma.

Esser, et al. [21] divided 29 mice with prostate cancer cells between two groups: Exercise group with 20 mice, and control group with nine mice. The exercise group engaged in a voluntary method of wheel running, while the control group performed no exercise. Research was conducted in 10 weeks. Among the running group, there was another division: Those who ran five $\mathrm{km}$ a day, and those who ran less than five $\mathrm{km}$. It was revealed that within those who ran five $\mathrm{km}$ a day, $83 \%$ of the dorsolateral prostates had been classified as within normal levels compared to $43 \%$ within the group that never ran beyond five $\mathrm{km}$. Furthermore, an association was drawn between average running distance and pathological progression. Forty-three percent of the dorsolateral prostates of mice which ran less than five $\mathrm{km}$ a day were classified as pathologically advanced compared to the mice which ran more, whose incidence of advanced pathology was null.

Antonelli, et al. [8] in a similar study involving mice in voluntary wheels, in which, a week before, subcutaneous, prostatic, tumor cells were applied, prompted researchers to discover that running decreases mitosis while increasing apoptosis on an underlying mechanistic basis from this growth inhibition.

Researchers further mention a study involving mice that revealed opposite results, which is to say, mice, which exercised, displayed an increase of prostate cancer xenograph growth and decreased rate of survival in the histological exam, $70 \%$ less necrosis, and $154 \%$ increase of microvascular density in endothelial structures.

\section{Possible mechanisms}

Several studies suggest the possible mechanisms which could likely justify how exercise influences possible protective associations against prostate cancer.

Ornish, et al. [7] as previously stated, show an increase in telomerase activity in human immune system cells within three months of lifestyle changes. In his review, Jaeger [22] suggests exercise may adjust the levels of blood testosterone and immune function and reduce serum levels of the free fraction of IGF-1.

Acute aerobic exercise increases testosterone levels, but the basal, total, and free levels are much lower among those who have been trained. Following the exercise is an increase in the levels of interleukina-1, 
interferons, and tumor necrosis factor alpha, increasing T lymphocyte activity, which in active individuals, will remain active as well, even when the individual is resting.

Johnsen, et al. [14] describe that certain observational studies were conducted, which provide some initial hints regarding molecular mechanisms with which physical exercise may inhibit prostate cell cancer. It changes the IGF axis increasing the cellular levels of the P53 protein, a DNA regulator which protects the genome through activating the cellular cycle detention, cause DNA to be repaired and/or undergo apoptosis when there are mutations in the genome.

Blood tests on men who participated in a regular exercise program for more than 10 years had lower IGF-1 and higher IGFBP-1, compared to sedentary men, and both changes increased the P53 content, reduced growth rate, and instigated apoptosis in cancer cells in vitro significantly higher degrees than in sedentary individuals. Moreover, through modulation of metabolic pathways, there are yet other molecular pathways, such as the antioxidant pathway and immune function, through which exercise could affect the biology of prostate cancer, and even improve energy balance. Chronic exercise has been shown as a possible modifier of the oxidant protection endogenous paths, decreasing lipid peroxidation and reactive species oxygen. One subjacent mechanism related to age responsible for the increase in the likelihood of prostate cancer is oxidative damage, including superoxide, hydrogen peroxide, and radical hidroxila.

Moore, et al. [15] describe the likely biological reasons why physical activity may decrease the risk of prostate cancer in black male. They are 20 times more likely to develop prostate cancer, have higher numbers of genes associated with inflammation and immune function, higher levels of testosterone during early adulthood, and lower resting energy expenditure (between 50 and $160 \mathrm{Kcal})$, compared to white men.

Galvão, et al. [23] showed a difference in reactive protein $\mathrm{C}$ between the groups after a 12-week program, which combined aerobic, and resistance exercises. The protein had lower values for the exercise group, and increased values for the control group. This is an important discovery, as low-degree systemic inflammation in elderly individuals is associated with several chronic conditions, as well as worsened physical performance and increased likelihood of mortality.

Richman, et al. [16] inform vigorous physical activity is associated with lower insulin, IGF-1, and inflammatory cytokines.

Shahar, et al. [17] also mentioned four mechanisms, which could explain the influence of physical activity in decreasing the risk of prostate cancer: Changes in endogenous hormones (lowered), decrease in body fat, improved immune function, and improved antioxidant defense mechanisms.

Galvão, et al. [24] report the increase of $\mathrm{GH}$ and DHEA may be partly the basis for the improvement observed in resistance training.

Heitkamp, et al. [19] noted physically active men have lower concentrations of testosterone and calcium in blood, with higher concentrations of vitamin $D$ and parathormones, as well improved antioxidant activities.

Khan, et al. [9] describe that obesity is yet another factor that may increase the risks of the causes of prostate cancer. Schlienger, et al. [25] further report that obesity is a public health issue with increased likelihood of developing cancer. The causal mechanisms remain unexplained, but epidemiological data and data collected from model animals have provided certain evidence of hormonal changes associated with obesity.

Both studies mention hyperinsulinemia and diabetes, high levels (or bioavailability) of IGF-1, increase in leptin levels, low levels adiponectin, high levels of estradiol serum (stemming from a reinforcement of aromatase), and all of which are related to the mitogenetic and anti-apoptotic effects of cancer cells. Obesity was associated with a worsened tumor prognosis and decreased likelihood of survival, particularly among black male.

\section{PSA and Exercise}

Ornish, et al. [6] show a decrease of $4 \%$ in the PSA for the lifestyle-changing group and an increase of $6 \%$ in the control group.

Moore, et al. [15] concluded sedentary individuals who do not engage in physical activities frequently higher concentrations of PSA compared to their more active peers.

Jaeger [22] states the effects of physical activities on PSA are controversial, but also no changes have been reported in most studies. Among the studies reporting changes, one demonstrates three times increase in base values among older men, and it is worth noting that the base PSA was already higher than normal. Another classic case study was conducted focused on a 70-year-old prostate cancer patient with $3244 \mathrm{ng} . \mathrm{mL}$ PSA. The study demonstrate the PSA decreased to $5.6 \mathrm{ng} . \mathrm{mL}$, upon the patient's interruption of his cycling exercises.

\section{The benefits of physical exercise for patients in treatment}

Galvão, et al. [26], Lee, et al. [27], Antonelli, et al. [8], Culos-Reed, et al. [11], Hansen, et al. [28], Alt, et al. [29] mentioned one third of men with prostate cancer develop the disease recurrently or metastatically, making it necessary to introduce other forms of treatment. It includes androgen deprivation therapy, orchiectomy, radiotherapy, and chemotherapy. Androgen deprivation 
therapy and orchiectomy aim to interrupt the production of testosterone, drastically slowing down the tumor's progression. However, a series of collateral effects result from these interventions, such as reduced libido, osteoporosis, weakened physical capabilities, faster onset of fatigue, loss of lean body mass, increased body fat and deteriorated psychosocial and cognitive functions. Recent data associated with deprivation therapy show an increased risk of cardiovascular morbidity and mortality, including new cases of diabetes, coronary artery disease, and sudden cardiac death.

Alt, et al. [29] studied the effects of resistance training twice a week for 20 weeks.

Hansen, et al. [28] assessed the effects of eccentric training using a cycle ergometer more often, with sessions between 12 and 15 minutes classified as "Somewhat Hard" in the Borg rating of perceived exertion (RPE) Scale. The researchers checked for benefits for prostate cancer patients undergoing androgen deprivation therapy and for those who did not receive such treatment.

Antonelli, et al. [8] showed two studies which set out to verify the effects of exercise in controlling symptoms, quality of life and physical aptitude in men receiving androgen deprivation treatment. In the first study, the active group engaged in resistance exercises three times a week. The second one was random, involving men with prostate cancer undergoing radiotherapy. The active group exercised for a period of six months.

Truong, et al. [30] presented a study in which prostate cancer patients undergoing radiotherapy walked with moderate intensity (60 to $70 \%$ heart rate) three times a week for 12 weeks.

Schneider, et al. [12] studied patients with prostate and mammary cancer under treatment (chemotherapy, radiotherapy, or surgery) engaging in exercises for six months, two to three times a week, with 10 to $15 \mathrm{~min}$ utes of warmup, 40 minutes of aerobic and resistance exercises involving two to three series of 8 to 12 repetitions, plus a 10-minute cool down.

The results of the aforementioned studies revealed improvements in collateral effects and morbidities caused by androgen deprivation, chemotherapy, radiotherapy, or surgery. There were also improvements in general quality of life, decrease in the incidence of fatigue, increase in strength and muscular resistance, improvements in cardiovascular conditioning, increased $\mathrm{GH}$ and DHEA, increase in lean body mass, reduce of triglycerides and body fat, better functional performance, balance, and, finally, improvements in depression.

\section{Physical exercise before surgery}

Lacza, et al. [31] show high levels of $\mathrm{VO}_{2} \max$ can prevent cardiovascular diseases, cancer, and neurodegenerative diseases, improving functioning in several organs.
Antonelli, et al. [8] mention a cohort study with patient's subject to surgical resection of malignant liver tumors. In this study, researchers found improvements in cardiorespiratory aptitude (increase of $2.4 \mathrm{ml} / \mathrm{kg} / \mathrm{min}$ ) seen in four to six weeks of training prior to surgery. Various studies investigating patients who underwent heart surgery showed the complications are inversely associated with physical aptitude.

Like these surgeries, could it be possible that improving prostate cancer patients' physical capabilities, acutely, prior to prostatectomy, may have similar benefits, reducing the incidence of complications following surgery?

Aside from likely reducing short-term morbidity and mortality stemming from surgery, physical exercise may lead to a long-term significant decrease in morbidity seen in prostatectomy and erectile dysfunction, with an estimated frequency fluctuating between $14 \%$ and $80 \%$. Physical exercise may theoretically improve erectile dysfunction following prostatectomy, due to modulating molecular mechanisms of nitric oxide, responsible for erection.

Exercise also influences myocardial function and the peripheral flow in the vascular endothelium, helping to reduce the risk of heart diseases.

\section{Recommendations}

Antonelli, et al. [8] conclude that these studies did not distinguish the type of physical activity, and few validated exposures with objective aptitude measures. Despite the fact that there is epidemiological data which provide promising evidence of exercise as capable of decreasing the risk of prostate cancer, studies which quantify physical activity with objective measures for determining a better, more truthful, associations must be conducted.

According to the American Cancer Society and the Prostate Cancer Foundation (as mentioned by Blanchard, et al. [13], Ottenbacher, et al. [32] and Antonelli, et al. [8]) recommend all prostate cancer patients must adopt a regular exercise program consisting of at least 20 minutes of aerobic exercises, and at least 5 days a week of moderately intense exercises. The program should also consist of resistance exercises two to three times a week for the key muscle groups, with two to three series of 8 to 12 repetitions of moderate intensity.

Galvão, et al. [26] further suggest 30 minutes of vigorous physical activities five times a week.

Schneider, et al. [12] recommend the volume and intensity should be set according to the patient's health state or physical aptitude. For sedentary patients with health or aptitude problems, exercises should begin with $30 \%$ to $45 \%$ of the Reserve Heart Rate, with one to three series of eight to twelve progressive and slowpaced repetitions. For active, moderately healthy pa- 
tients with average aptitude, begin with $50 \%$ to $60 \%$ of the Reserve Heart rate, with four to five series of eight to twelve progressive, slow-paced repetitions.

Despite recommendations from the aforementioned institutes, Blanchard, et al. [13] show that only $29.6 \%$ to $47.3 \%$ of men with prostate cancer engage in physical activities.

Culos-Reed, et al. [11], and Liu, et al. [18] state patients with prostate cancer must be encouraged by doctors to exercise. Keogh, et al. [33] show that $70 \%$ of patients were not advised about exercise and its benefits during treatment.

According to Baumann, et al. [34] when prescribing exercises for prostate cancer patients, it is necessary to consider aspects related to the disease, such as urinary incontinence, erectile dysfunction, fatigue, fear, and depression, which may lead the patient into isolation from social life.

\section{Conclusion}

Quality of life has been improved in patients with prostate cancer when one's lifestyle undergoes changes, including when subject to deprivation therapy, chemotherapy, and radiotherapy. However, despite promising epidemiological evidence strongly suggesting that moderate to vigorous exercise may impact prevention, delaying, and progression of prostate cancer, no associations between exercise and prostate cancer have been confirmed. Further studies quantifying and controlling exercise and the mechanisms involved in the process are required.

\section{Acknowledgement}

The present study was financially supported by CAPES.

\section{References}

1. Tonon TCA, Schoffen JPF (2009) Câncer de prostata: uma revisão de literatura. Revista Saúde e Pesquisa 2: 403-410.

2. (2014) Instituto Nacional de Câncer (INCA) 2014.

3. Schreiber DS (2007) Anticâncer. Prevenir e vencer usando nossas defesas naturais. ( $1^{\text {st }}$ edn), São Paulo, Fontanar.

4. Frattaroli J, Weidner G, Dnistrian AM, Kemp C, Daubenmier $\mathrm{JJ}$, et al. (2008) Clinical events in prostate cancer lifestyle trial: Results from two years of follow-up. Urology 72: 1319-1323.

5. Daubenmier JJ, Weidner G, Marlin R, Crutchfield L, Dunn-Emke S, et al. (2006) Lifestyle and health-related quality of life of men with prostate cancer managed with active surveillance. Urology 67: 125-130.

6. Ornish D, Weidner G, Fair WR, Marlin R, Pettengill EB, et al. (2005) Intensive lifestyle changes may affect the progression of prostate cancer. J Urol 174: 1065-1069.

7. Ornish D, Lin J, Chan JM, Epel E, Kemp C, et al. (2013) Effect of comprehensive lifestyle changes on telomerase activity and telomere length in men with biopsy-proven lowrisk prostate cancer: 5-year follow-up of a descriptive pilot study. Lancet Oncol 14: 1112-1120.
8. Antonelli J, Freedland SJ, Jones LW (2009) Exercise therapy across the prostate cancer continuum. Prostate Cancer Prostatic Dis 12: 110-115.

9. Khan N, Afaq F, Mukhtar H (2010) Lifestyle as risk factor for cancer: Evidence from human studies. Cancer Lett 293: 133-143.

10. Sheffield J, Wood EF, Roderick ML (2012) Little change in global drought over the past 60 years. Nature 491: 435-438.

11. Culos-Reed SN, Robinson JL, Lau H, O Connor K, Keats MR (2007) Benefits of physical activity intervention for men with prostate cancer. J Sport Exerc Psychol 29: 118-127.

12. Schneider CM, Hsieh CC, Sprod LK, Carter SD, Hayward $R$ (2007) Cancer treatment-induced alterations in muscular fitness and quality of life: The role of exercise training. Ann Oncol 18: 1957-1962.

13. Blanchard CM, Courneya KS, Stein K (2008) Cancer survivor's adherence to lifestyle behavior recommendations and associations with health-related quality of life: Results from the American cancer Society's SCS-II. J Clin Oncol 26: 2198-2204.

14. Johnsen NF, Tionneland A, Thomsen BL, Christensen J, Friedenreich C, et al. (2009) Physical activity and risk of prostate cancer in the European Prospective Investigation into Cancer and Nutrition (EPIC) cohort. Int J Cancer 125: 902-908.

15. Moore SC, Peters TM, Ahn J, Park Y, Schatzkin A, et al. (2009) Age-specific physical activity and prostate cancer risk among white men and black men. Cancer 115: 5060-5070.

16. Richmann EL, Kenfield SAS, Tampfer MJ, Paciorek A, Carroll PR, et al. (2011) Physical activity after diagnosis and risk of prostate cancer progression: Data from the cancer of the prostate strategic urologic research endeavor. Cancer Res 71: 3889-3895.

17. Shahar S, Shafurah S, Hasan NS, Rajikan R, Rajab NF, et al. (2011) Roles of diet, lifetime physial activity and oxidative DNA damage in the occurrence of prostate cancer among men in Klang Valley, Malasya. Asian Pac J Cancer Prev 12: 605-611.

18. Liu Y, Hu F, Li D, Wang F, Zhu L, et al. (2011) Does physical activity reduce the risk of prostate cancer? A systematic review and meta-analysis. Eur Urol 60: 1029-1044.

19. Heitkamp HC, Jelas I (2012) Physical activity for primary prevention of prostate cancer. Possibles mechanisms. Urologe A 51: 527-532.

20. Crespo CJ, Garcia-Palmiere MR, Smit E, Lee IM, Mcgee D, et al. (2008) Physical activity and prostate câncer mortality in puerto rican men. J Phys Act Health 5: 918-929.

21. Esser KA, Harpole CE, Prins GS, Diamond AM (2009) Physical activity reduces prostate carcinogenesis in a transgenic model. Prostate 69: 1372-1377.

22. Jaeger CDO (2008) O efeito do exercício físico em esteira rolante nos níveis séricos do antígeno prostático específico. Dissertação de Mestrado. Porto Alegre. Universidade Federal do Rio Grande do Sul.

23. Galvão DA, Taaffe DR, Spry N, Joseph D, Newton RU (2010) Combined resistance and aerobic exercise program reverses muscles loss in men undergoing androgen suppression therapy for prostate cancer without bone metastases: A randomized controlled trial. J Clin Oncol 28: 340-347.

24. Galvão DA, Nosaka K, Taaffe DR, Peake J, Spry N, et al. (2008) Endocrine and immune responses to resistance training in prostate cancer patients. Prostate Cancer Prostatic Dis 11: 160-165. 
25. Schlienger JL, Lca F, Vinzio S, Pradignac A (2009) Obesity and câncer. Rev Med Interne 30: 776-782.

26. Galvão DA, Spry N, Taaffe DR, Denham J, Joseph D, et al. (2009) A randomized controlled trial of an exercise targeting cardiovascular and metabolic risk factors for prostate cancer patients from the RADAR trial. BMC Cancer 9: 419.

27. Lee CE, Lelie WD, Lau YK (2012) A pilot study of exercise in men with prostate cancer receiving androgen deprivation therapy. BMC Cancer 12: 103.

28. Hansen PA, Dechet CB, Porucznik CA, LaSatyo PC (2009) Comparing eccentric resistance exercise in prostate cancer survivors on and off hormone therapy: A pilot study. PM R 1: $1019-1024$

29. Alt CA, Gore EM, Montagnini ML, Ng AV (2011) Muscle endurance, cancer-related fatigue and radiotherapy and prostate cancer survivor. Muscle Nerve 43: 415-424.

30. Truong PT, Gaul CA, McDonald RE, Petersen RB, Jones
SO, et al. (2011) Prospective evaluation of a 12-week walking exercise program and its effect on fatigue in prostate cancer patients undergoing radical external beam radiotherapy. Am J Clin Oncol 34: 350-355.

31. Lacza G, Radák Z (2013) Is physical activity an elixir? Orv Hetil 154: 764-768.

32. Ottenbacher AJ, Day RS, Taylor WC, Sharma SV, Sloane $\mathrm{R}$, et al. (2011) Exercise among breast and prostate cancer survivor. What are their barriers? J Cancer Surviv 5: 413419.

33. Keogh JW, Macleod RD (2012) Body composition, physical fitness, functional performance, quality of life and fatigue benefits of exercise for prostate cancer patients: A systematic review. J Pain Symptom Manage 43: 96-110.

34. Baumann FT, Zopf EM, Block W (2012) Clinical exercises interventions in prostate cancer patients - a systematic review of randomized controlled trials. Support Care Cancer 20: 221-233. 